\title{
Nanosensors for Monitoring Early Stages of Metallic Corrosion
}

\author{
Loïc Exbrayat, ${ }^{\S, \perp, \#}$ Suttiruk Salaluk, ${ }^{\dagger, \#}$ Matthias Uebel, ${ }^{\ddagger}$ (৫) Ratchapol Jenjob, ${ }^{\dagger}$ Bruno Rameau,

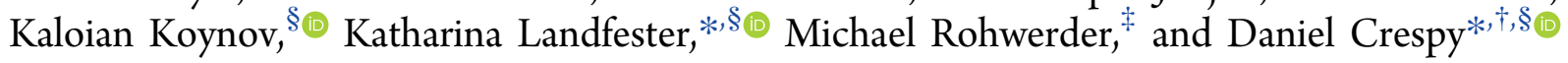

${ }^{\dagger}$ Vidyasirimedhi Institute of Science and Technology (VISTEC), Rayong 21210, Thailand

${ }^{\ddagger}$ Max-Planck-Institut für Eisenforschung GmbH, 40237 Düsseldorf, Germany

${ }^{\S}$ Max Planck Institute for Polymer Research, 55128 Mainz, Germany

"ArianeGroup, Avenue du Général Niox, 33165 Saint-Médard-en Jalles, France

${ }^{\perp}$ LaSIE, Université de la Rochelle, Avenue Michel Crépeau, F-17042 La Rochelle, France

Supporting Information

ABSTRACT: A new generation of nanosensors based on mesoporous silica nanocapsules with the ability to monitor the onset of metallic corrosion is successfully developed and tested on 304 stainless steel. The core of the nanocapsules contains water insoluble organic molecules that fluoresce during the anodic dissolution of metallic substrates in the corrosion process. The dispersion of the nanosensors in organic coatings applied on metal substrate allows a very sensitive fluorescent detection of the initiation of metal dissolution, close to defects in the substrate. This promising concept offers therefore new perspectives for the development of smart coatings for corrosion sensing.

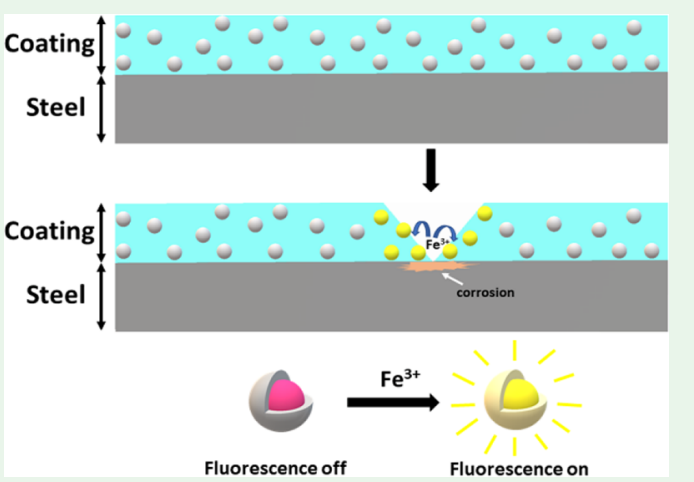

KEYWORDS: corrosion sensing, nanosensor, silica nanocapsules, smart coating, turn-on fluorescence

\section{INTRODUCTION}

The corrosion of metals is a critical issue that has globally huge impacts in in terms of safety and economy. Corrosion of metals has a huge impact in the construction, aerospace, and automotive industries since the deterioration of metallic components impacts the life cycle of structures. Many structures such as ships, aircrafts, and car bodies are manufactured from a wide range of metals such as carbon steels or aluminum and are usually protected from corrosion by primers and organic coatings. During service, coatings can be deteriorated and anodically active areas are formed, which may lead to relatively fast delamination of the painted areas. Therefore, an early detection of corrosion is of significant interest in economic, safety, and ecological scales. Different physical methods are commonly used to detect corrosion but have the drawback that active external handling is necessary to obtain information about corrosion. Therefore, smart coatings were developed so that they provide feedback on the corrosion state of underlying metals. A first approach is to embed a dye in the coating that is sensitive to changes of environmental $\mathrm{pH}$ values. First examples were coatings composed of one sensing layer sandwiched between two layers of glass mat/unsaturated polyester resin layer. ${ }^{1}$ The $\mathrm{pH}$-sensitive phenolphthalein was directly mixed in the coating layer and was found to give a purple color to the laminate when it was immersed in sodium hydroxide solutions. Similarly, when a phenanthroline dye was added in an alkyd resin ${ }^{2}$ or acrylic coating ${ }^{3}$ on mild steel, a color change could be observed because of the complexation of the dye with $\mathrm{Fe}^{2+}$. In order to protect the dye from detrimental interactions with the coating matrix, $\mathrm{pH}$ indicators can be encapsulated. Micro- and nanoencapsulation have also the advantage to increase locally the concentration of the dye so that a more intense color change is observed. The $\mathrm{pH}$ indicator phenolphthalein, which changes color at high $\mathrm{pH}$ values, was encapsulated in mesoporous nanoparticles by taking advantage of an optical change at active cathodic zones ${ }^{4}$ in aluminum and magnesium alloys. A similar approach was used with polyurea microcapsules. ${ }^{5}$ However, the approaches based on color change suffered from the fact that corrosion products are usually already colored and that they are not effective at the early stage of corrosion.

To tackle this issue, fluorescence sensors, which are much more sensitive, have been used widely for detecting either changes of the $\mathrm{pH}$ value or the presence of metal cation due to corrosion. ${ }^{6,7}$ This approach was applied by mixing fluorescent dyes sensitive to changes of $\mathrm{pH}$ values and redox conditions into primer coatings for aluminum. ${ }^{8}$ Fluorescent dyes were used also to label aluminum alloy surfaces to detect local $\mathrm{pH}$ changes by fluorescence microscopy. ${ }^{9}$ One option to detect $\mathrm{pH}$ change is to couple a $\mathrm{pH}$-sensitive dye or a polymer-dye

Received: November 13, 2018

Accepted: January 11, 2019

Published: January 11, 2019 
conjugate to optical fibers. Internal holes of microstructured fibers were filled with a dye attached to a polymer for the detection of aluminum ions by fluorescence. ${ }^{10}$ Similarly, different dyes were immobilized on optical fibers for detecting by fluorescence low and high $\mathrm{pH}$ values or aluminum ions ${ }^{11}$ in the environment of aluminum surfaces. ${ }^{12}$ Coumarin imidazole was modified to afford a polymerizable dye that was copolymerized with methacrylic acid and 1,4-bis(acryloyl)piperazine to yield a cross-link gel that was fixed at the end of an optical fiber. ${ }^{13}$ The dye was fluorescent when protonated $\left(\mathrm{p} K_{\mathrm{a}}=12.5\right)$, while it was less fluorescent when it was deprotonated due to photoinduced electron transfer between the receptor and the fluorophore. The fiber was used to detect corrosion in concrete and was active for $\mathrm{pH}$ values between 10 and 13. The presence of iron ions was detected during steel corrosion by embedding 8 -hydroxyquinoline ${ }^{14}$ or a fluorescent dye derived from rhodamine ${ }^{15}$ in epoxy, which both was a corrosion inhibitor and served as fluorescent sensor upon complexation with iron ions. Microcapsules were also used in thiol-epoxy thermosets in order to provide materials that can respond both to corrosion and mechanical damage. ${ }^{16}$ While the $\mathrm{pH}$ indicator thymol blue was embedded directly in the epoxy matrix, tetraphenylethylene dissolved in hexyl acetate was physically entrapped in microcapsules. Tetraphenylethylene displayed an aggregation-induced emission when cracks occurred after evaporation of the solvent hexyl acetate.

Herein, we present a coating containing nanosensors that are able to detect iron ions and low $\mathrm{pH}$ values induced by the corrosion of steel substrates. The nanosensors consist of a mesoporous silica nanocontainer with a hydrophobic liquid core containing a dye. The dye was selected because it displays chelation-enhanced fluorescence in the presence of iron(III) ions. This design impedes premature leaking of the dye due to the hydrophobic nature of the core while allowing protons and iron ions to access the interior of the core so that a strong fluorescence signal can be detected.

\section{EXPERIMENTAL SECTION}

2.1. Materials. Tetraethyl orthosilicate (TEOS, Acros Organics, 98\%), $n$-hexadecane (HD, Acros Organics, 99\%), cetyltrimethylammonium chloride (CTMA-Cl, TCI, 95\%), cetyltrimethylammonium bromide (CTAB, Acros Organics, 99\%), acetonitrile (Carlo Erba), rhodamine B (Merck), toluene (Carlo Erba), ethanol (Carlo Erba), acetone (Acros Organics, 98\%), dichloromethane (Honeywell, 99.9\%), hydrochloric acid (Carlo Erba, 37\%), iron(III) chloride anhydrous $\left(\mathrm{FeCl}_{3}\right.$, Carlo Erba), ammonium iron(II) sulfate hexahydrate $\left(\left(\mathrm{NH}_{4}\right)_{2} \mathrm{Fe}\left(\mathrm{SO}_{4}\right)_{2} \cdot 6 \mathrm{H}_{2} \mathrm{O}\right.$, Merck $)$, zinc chloride anhydrous $\left(\mathrm{ZnCl}_{2}\right.$, Carlo Erba), aluminum chloride $\left(\mathrm{AlCl}_{3}\right.$, Acros Organics), sodium chloride ( $\mathrm{NaCl}$, Carlo Erba), and hydrazine monohydrate (TCI, 98\%) were used as received. All chemicals were analytic grade and were used without further purification. The dyes for metallic ions sensing were synthesized according to literature procedures. ${ }^{17}$

2.2. Design of the Nanosensors. A known amount of dye was dissolved in $2 \mathrm{~g}$ of TEOS, $125 \mathrm{mg}$ of HD, and $1 \mathrm{~g}$ of toluene. The mixture was stirred with $30 \mathrm{~mL}$ of a 0.77 wt \% aqueous solution of CTMA-Cl. The emulsion was then sonicated under ice cooling for $180 \mathrm{~s}$ at $70 \%$ amplitude in a pulse regime (30 s sonication, $10 \mathrm{~s}$ pause) using a Branson $450 \mathrm{~W}$ sonifier and a $1 / 2$ in. tip. The resulting emulsion was then further stirred $20 \mathrm{~h}$ to obtain the silica nanocapsules. For the fluorescence measurements, $15 \mathrm{~mL}$ of a 13.3 $\mathrm{mg} \mathrm{mL} L^{-1}$ aqueous solution of $\mathrm{CTAB}$ was used to avoid the presence of chloride ions.

2.3. Characterization of the Nanosensors. The sizes of silica nanocapsules were measured by dynamic light scattering (DLS,
NanoPlus, Particulate Systems). The surface morphology of silica nanocapsules was observed with a scanning electron microscope (SEM, JSM-7610F, JEOL). Samples dispersed at an appropriate concentration were cast onto a silicon wafer at room temperature. The morphology of silica nanocapsules was observed by transmission electron microscopy (TEM, JEM-ARM 200F, JEOL) at an acceleration voltage of $200 \mathrm{kV}$. Samples dispersed at an appropriate concentration were cast onto a carbon-coated copper grid. The average size of silica nanocapsules was determined by counting 100 particles with the SemAfore software 5.21 (JEOL). For UV spectroscopy investigations, stock solutions of $\mathrm{Fe}^{3+}$ were prepared using $\mathrm{FeCl}_{3}$ in acetonitrile:water $(1: 1, \mathrm{v}: \mathrm{v})$ or water. Solutions of rhodamine derivative and silica nanocapsules with encapsulated dye for spectroscopic studies were also prepared in acetonitrile:water $(1: 1$, v:v) or water. UV absorption (Lambda 1050, PerkinElmer) and fluorescence (FLS980, Edinburgh Instruments) studies were performed using $25 \mu \mathrm{mol} \mathrm{L} \mathrm{L}^{-1}$ and $50 \mu \mathrm{mol} \mathrm{L^{-1 }}$ solutions of rhodamine derivative and silica nanocapsules with encapsulated rhodamine derivative ( $0.030 \mathrm{wt} \%$ dye in the whole dispersion) in appropriate amounts of $\mathrm{Fe}^{3+}$ ions. The solutions were shaken for $6 \mathrm{~h}$ before measuring the absorption and fluorescence. The excitation was performed at $510 \mathrm{~nm}$ for all the emission studies. Encapsulation efficiency was estimated by UV-visible spectroscopy. First, a known amount of the miniemulsion $(0.5 \mathrm{~mL})$ was mixed with the same volume of $35 \mathrm{~g} \mathrm{~L}^{-1} \mathrm{NaCl}$ solution and centrifuged to separate the capsules from the solution. The capsules were then dissolved in $5 \mathrm{~mL}$ of dichloromethane during $48 \mathrm{~h}$ to extract the dye from the core of the capsule. The amount of dye extracted was then measured by UVvisible spectroscopy according to a preliminary calibration at $317 \mathrm{~nm}$, and compared to the theoretical amount. The ratio corresponds to the encapsulation efficiency. Fluorescence correlation spectroscopy (FCS) experiments were performed with a commercial setup (Carl Zeiss, Germany) consisting of the modules ConfoCor 2 and LSM 510 coupled to an inverted microscope (Axiovert 200), equipped with a Zeiss C-Apochromat $40 \times /(1.2 \mathrm{~W})$ water immersion objective. For excitation, the $543 \mathrm{~nm}$ line of a HeNe laser was used, and collected fluorescence was filtered through a LP560 long pass emission filter. Eight-well polystyrene-chambered coverglass (Laboratory-Tek, Nalge Nunc International, Penfield, NY, USA) was used as a sample cell. FCS measurements were performed with nanosensors dispersed (factor of $1 / 60$ ) in four different dispersions: two dispersions in aqueous solutions of ferric ions with concentrations of $0.05 \mathrm{mmol} \mathrm{L}^{-1}$ and $5 \mathrm{mmol} \mathrm{L}^{-1}$ and two dispersions in pure water with $\mathrm{pH}$ adjusted by $\mathrm{HCl}$ addition to the same values as the aforementioned ones for ferric solutions (respectively $\mathrm{pH}=5$ and $\mathrm{pH}=2.9$ ). All dispersions were kept stirring, and FCS experiments were performed at different time intervals after the dispersions preparation. During an FCS experiment the time-dependent fluctuations of the fluorescence intensity $\delta I(t)$ caused by the diffusion of the fluorescent species (loaded nanocapsules or freely diffusing activated fluorescent molecules) through the confocal observation volume were recorded and analyzed by an autocorrelation function $G(t)=1+\langle\delta I(t) \delta I(t$ $+\tau)\rangle /\langle I(t)\rangle^{2}$. For an ensemble of $m$ different types of freely diffusing fluorescence species, $G(t)$ has the following analytical form. ${ }^{18}$

$$
G(t)=1+\frac{1}{N} \sum_{i=1}^{m} \frac{f_{i}}{\left[1+\frac{\tau}{\tau_{\mathrm{D} i}}\right] \sqrt{1+\frac{\tau}{S^{2} \tau_{\mathrm{D} i}}}}
$$

Here, $N$ is the average number of diffusing fluorescence species in the observation volume, $\tau_{D_{i}}$ is the diffusion time of the $i$ th species, $f_{i}$ is the fraction of component $i$, and $S$ is the so-called structure parameter, $S$ $=z_{0} / r_{0}$, where $z_{0}$ and $r_{0}$ represent the axial and radial dimensions of the confocal volume, respectively. Furthermore, the diffusion time, $\tau_{D_{i}}$, is related to the respective diffusion coefficient, $D_{i}$, through $^{18} D_{i}=r_{0}^{2} /$ $4 \tau_{D_{i}}$. The experimentally obtained $G(t)$ can be fitted with eq 1 yielding the corresponding diffusion times and subsequently the diffusion coefficients of the fluorescent species. The hydrodynamic radii, $R_{\mathrm{h}}$, can be calculated (assuming spherical particles) using the 


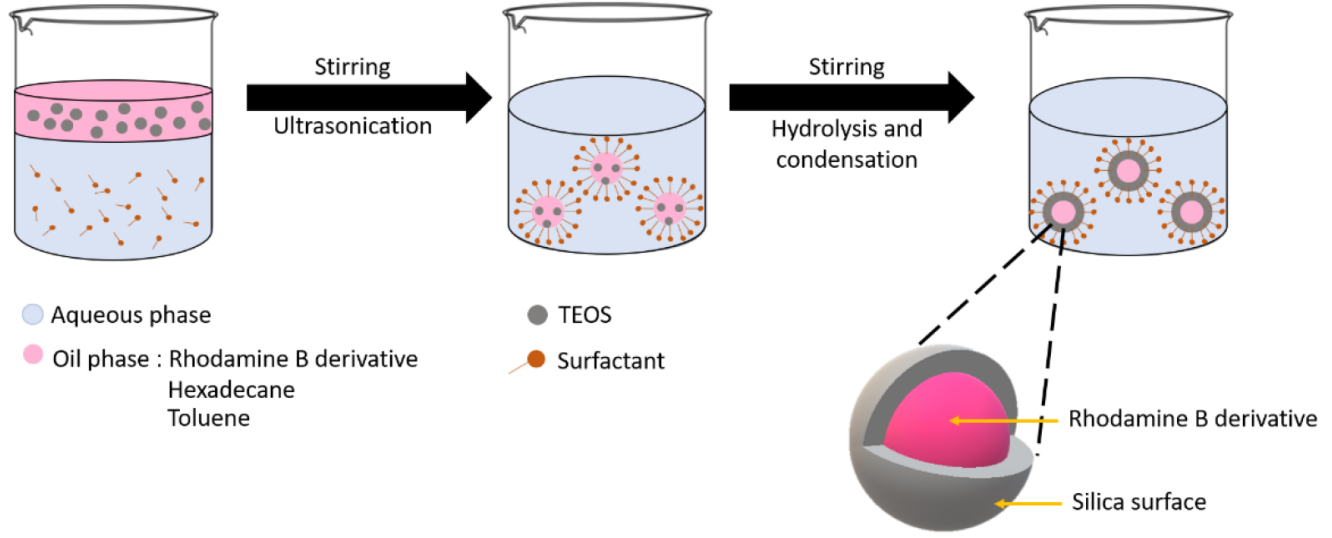

Figure 1. Schematics for the preparation of the nanosensors based on mesoporous silica nanocontainers.

Stokes-Einstein relation: $R_{\mathrm{h}}=k_{\mathrm{B}} T / 6 \pi \eta D$, where $k_{\mathrm{B}}$ is the Boltzmann constant, $T$ is the temperature, and $\eta$ is the viscosity of the solution. Furthermore, the fluorescent brightness of the species can be estimated by dividing the average fluorescent intensity to the average number of fluorescent species in the probing volume; i.e., $\mathrm{FB}=$ $\langle I(t)\rangle / N$.

2.4. Preparation of the Composite Coating. A $50 \mu \mathrm{L}$ aliquot of the nanosensor dispersion was dropped on the metal sample and spin-coated at $3000 \mathrm{rpm}$ for $20 \mathrm{~s}$. This operation was repeated three times. Then, 5 wt \% poly(vinyl butyral-co-vinyl alcohol-co-vinyl acetate) dissolved in ethanol was used as a binder and also spincoated three times at $2000 \mathrm{rpm}$ for $20 \mathrm{~s}$. This protocol led to the formation of polymer/nanosensors composite coating with a poly(vinyl butyral-co-vinyl alcohol-co-vinyl acetate) top coat. Based on previous experiments with the same coating, ${ }^{19}$ the thickness was evaluated to be $\sim 1.5 \mu \mathrm{m}$. Once the coating had been applied, it was allowed to dry for $1 \mathrm{~h}$ at room temperature.

2.5. Corrosion Experiment. A 304 stainless steel sample, mechanically polished with 1000 grade grinding paper and submitted to ultrasonication in ethanol, was coated according to the abovementioned protocol and used as a working electrode in a classical three-electrode electrochemical cell. The sample was affixed and sealed in order to have a $0.44 \mathrm{~cm}^{2}$ area exposed to the $35 \mathrm{~g} \mathrm{~L}^{-1} \mathrm{NaCl}$ solution. Platinum foil and $\mathrm{Ag} / \mathrm{AgCl}$ electrode were used as counter and reference electrodes. To initiate localized corrosion, the sample was submitted to a potentiodynamic polarization scan with a sweep rate of $2 \mathrm{mV} \mathrm{s}^{-1}$ until the corrosion density reached a threshold of about $2 \mathrm{~mA} \mathrm{~cm}^{-2}$. The sample was then investigated by means of a fluorescence microscope observation 1 week after the polarization experiments. Fluorescence imaging was conducted with an Olympus IX81 fluorescent microscope and CY3 filter $\left(\lambda_{\mathrm{ex}}=531 \mathrm{~nm}\right.$ and $\lambda_{\mathrm{em}}=$ $593 \mathrm{~nm}$ ) with a fixed gain of $18 \mathrm{~dB}$ and exposure time of $350 \mathrm{~ms}$.

\section{RESULTS AND DISCUSSION}

The nanosensors were fabricated as nanocontainers by a solgel process in miniemulsion depicted in Figure 1. Briefly, a synthesized rhodamine B derivative is dissolved in an organic phase consisting of an organic solvent and the silica precursor (alkoxysilane). The mixture is then emulsified by ultrasonication to form a miniemulsion with nanodroplets dispersed in water and stabilized by a surfactant. An interfacial reaction takes place when the alkoxysilane is hydrolyzed and then condensed to form a silica shell surrounding the solution of dye. This process was already reported for encapsulating selfhealing agents, ${ }^{20}$ a perylene dye, ${ }^{21}$ corrosion inhibitors, ${ }^{22,23}$ peppermint oil, ${ }^{24}$ or precursors for the formation of metal oxide catalysts. ${ }^{25}$ The gates of the pores, which control permeability of the ions, are not responsive to corrosion. The sol-gel process occurring at the interface of miniemulsion droplets was found to display porous structures as measured previously. $^{22}$ The noncomplexed dye solubility in water is extremely low, and therefore no significant transport of the dye to the aqueous phase is expected if the dye is not complexed.

After the hydrolysis and the condensation reactions, silica nanocapsules with an average hydrodynamic diameter depending on the surfactant concentration are formed, for example, hydrodynamic diameters of $55 \mathrm{~nm}(\mathrm{PDI}=0.19)$ and geometric diameters of $40 \pm 10$ and $43 \pm 10 \mathrm{~nm}$ as determined by DLS and SEM, respectively, with 7.4 wt \% surfactant compared to the dispersed phase. Spherical nanoparticles with a welldefined core-shell structure could be identified by electron microscopy (Figure 2). The encapsulation efficiency of the dye
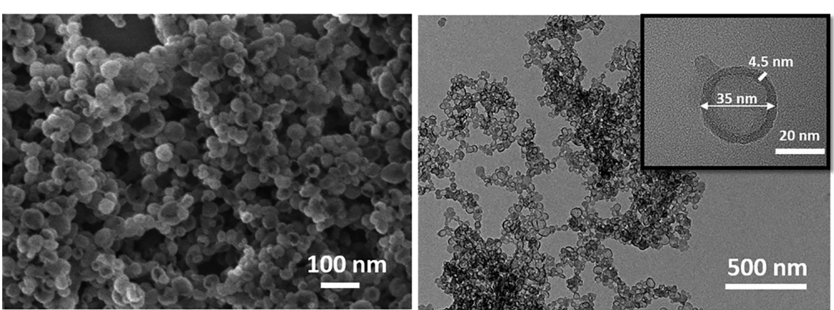

Figure 2. SEM micrograph (left) and TEM micrograph (right) of the silica nanocapsules.

inside the capsule was calculated to be $97 \%$ corresponding to a dye concentration of $17.46 \mathrm{mmol} \mathrm{L}^{-1}$ in toluene inside the core of the capsules.

The dye displayed strong fluorescence upon illumination with a UV lamp in the presence of $\mathrm{FeCl}_{3}$ (see Supporting Information Figure S1). The maximum absorbance was identified to be $560 \mathrm{~nm}$ (Figure S2), as reported in another report. ${ }^{15}$ The dye is only very weakly fluorescent in the absence of metal ions because fluorescence is quenched by photoinduced electron transfer effect. ${ }^{26}$ However, metal ion coordination with the lone electron pairs of nitrogen and oxygen of the dye brings the energy state of the lone pair below the HOMO of the dye so that fluorescence occurs (Figure S3). As expected, the fluorescence intensity of the dye solution was dependent on the dye concentration and on the amount of iron(III) ions present in the solution (Figure S4). Remarkably, strong fluorescence signals could be detected after encapsulation of the dye in the nanocontainers and the intensity was found to increase with $\mathrm{Fe}^{3+}$ concentration (Figure 3). To decouple the effect of chelation-induced fluorescence and the 

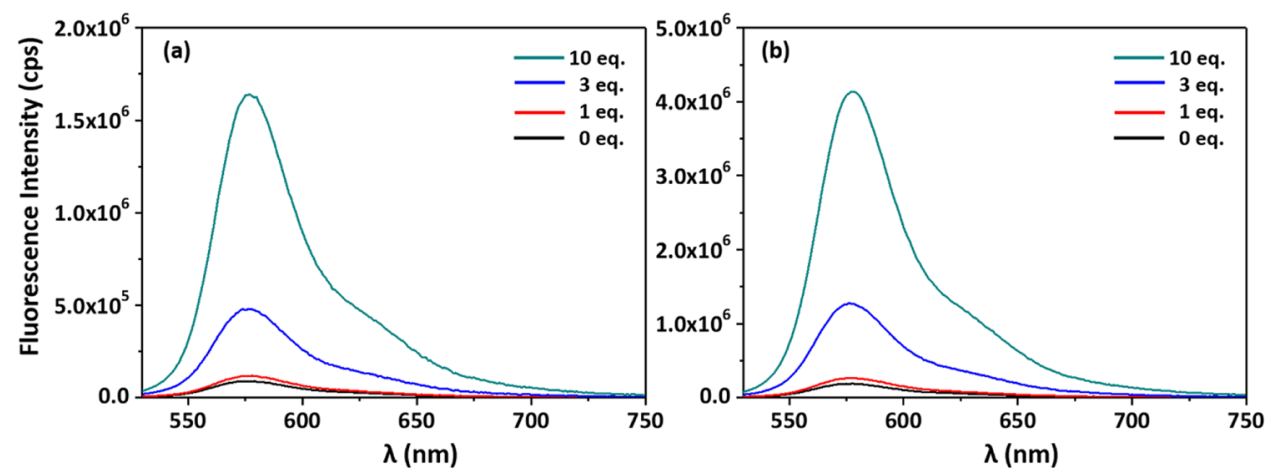

Figure 3. Fluorescence emission spectra of the dispersion of nanocontainers dispersed in water encapsulating a solution of dye ((a) 25 and (b) 50 $\mu$ mol L $\left.{ }^{-1}\right)$ excited at $510 \mathrm{~nm}$ upon addition of $0-10$ equiv of $\mathrm{Fe}^{3+}$ in acetonitrile: $\mathrm{H}_{2} \mathrm{O}(1: 1(\mathrm{v}: \mathrm{v}))$.
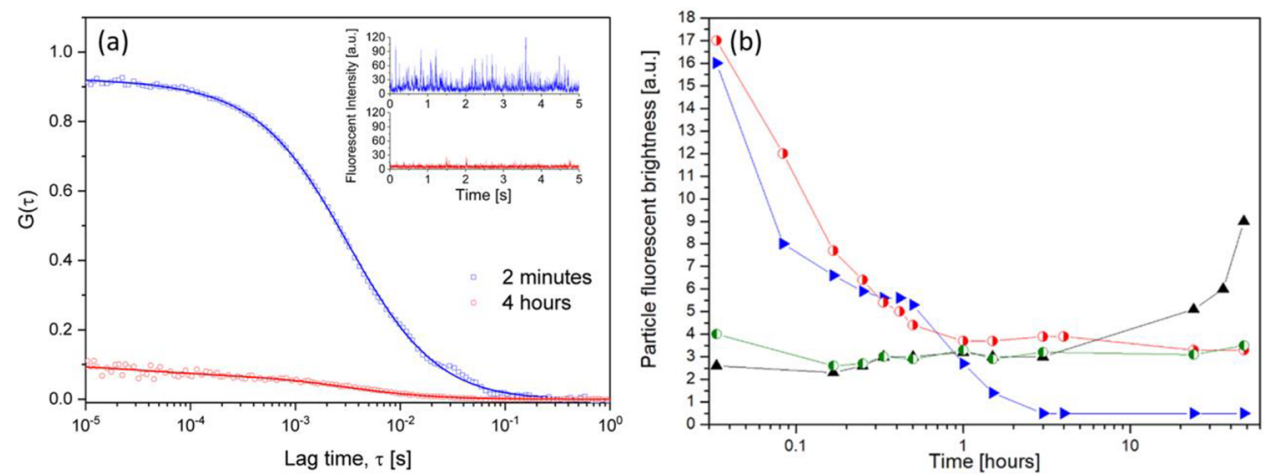

Figure 4. (a) Typical FCS autocorrelation curves measured $2 \mathrm{~min}(\square)$ and $4 \mathrm{~h}(\mathrm{O})$ after dispersing the nanocontainers in the solution with $5 \mathrm{mmol}$ $\mathrm{L}^{-1}$ ferric ions concentration. The solid lines represent fits with eq 1 . The inset shows the corresponding fluorescent intensity time traces. (b) Time evolution of the fluorescence brightness of nanocontainers dispersed in two different solutions of ferric ions (black line with triangles, $0.05 \mathrm{mmol}$ $\mathrm{L}^{-1}$; blue lines with triangles, $5 \mathrm{mmol} \mathrm{L}{ }^{-1}$ ) and two aqueous solutions adjusted to their corresponding pH values (green line with half-filled circles, $\mathrm{pH}=5$; red line with half-filled circles, $\mathrm{pH}=2.9$ ).

effect of the ions on the $\mathrm{pH}$ value, we investigated the fluorescence of the dye in the presence of various amounts of strong acids. Although the effect of $\mathrm{HCl}$ was markedly less significant than the effect of $\mathrm{FeCl}_{3}$ on fluorescence, a trend could be observed (Figure S5). Indeed, the fluorescence intensity increased with the concentration of the acid. A similar trend was observed for the dispersion of nanosensors (Figure S6). The nanosensors were sensitive to a very low amount (sensitivity of $\sim 10 \mu \mathrm{M}$ ) of iron(III) ions, rendering them ideal for detection of early stages of corrosion of steel substrates). Indeed, typical concentrations close to the corroding surface are usually in the micromolar range. ${ }^{27}$

It is of interest to determine the localization of the fluorescent species after activation of the nanosensors. Therefore, we verified whether the activated fluorescent molecules were still encapsulated in the nanocapsules or freely diffusing in the aqueous environment. We followed the temporal evolution of the fluorescent behavior of nanosensors dispersions upon addition of acidic solutions in the presence or absence of ferric ions (Figure 4) by fluorescence correlation spectroscopy (FCS). ${ }^{28}$ In a FCS experiment, the fluorescence intensity fluctuations caused by the diffusion of fluorescent species through a very small observation volume (the focus of a confocal microscope) are monitored. Correlation analysis of these fluctuations yields information on the hydrodynamic radius, their concentration, and their fluorescent brightness ${ }^{29}$ (see Experimental Section for details). The FCS measurements were performed with the nanosensors dispersed in four different environments: two dispersions in aqueous solutions of ferric ions with concentrations of $0.05 \mathrm{mmol} \mathrm{L}^{-1}$ and 5 mmol $\mathrm{L}^{-1}$ and two dispersions in pure water with a $\mathrm{pH}$ value adjusted by addition of $\mathrm{HCl}$ to the same values as the one of the corresponding ferric solutions (respectively $\mathrm{pH}=5$ and $\mathrm{pH}$ $=2.9$ ).

Typical autocorrelation curves measured respectively $2 \mathrm{~min}$ and $4 \mathrm{~h}$ after dispersing the nanocontainers in the solution with the highest concentration of ferric ions $\left(5 \mathrm{mmol} \mathrm{L}^{-1}\right)$ are shown in Figure $4 \mathrm{a}$. The curve measured after 2 min can be well-fitted with eq 1 using one component only $(m=1$; see Experimental Section), yielding a value of $R_{\mathrm{h}}=58 \pm 5 \mathrm{~nm}$ for the hydrodynamic radius of the fluorescent species present in the dispersions. This value is consistent with the size of the nanocontainers. It indicated that, shortly after mixing of the nanocontainers and the ferric ions, the fluorescent activated molecules were formed and remained in the nanocontainers. On the other hand, the autocorrelation curve measured after 4 $\mathrm{h}$ had to be fitted with two components $(m=2$; see Experimental Section): the first component with a hydrodynamic radius of around $58 \mathrm{~nm}$ and the second one with a hydrodynamic radius of less than $1 \mathrm{~nm}$. This result indicated that a significant fraction of the activated fluorescent molecules was no longer encapsulated in the nanocontainers after $4 \mathrm{~h}$, but was released and freely diffusing in the aqueous environment. This is further illustrated by the corresponding time traces of the measured fluorescence intensity fluctuation shown in the inset of Figure $4 \mathrm{a}$. On the trace measured 2 min after mixing, 

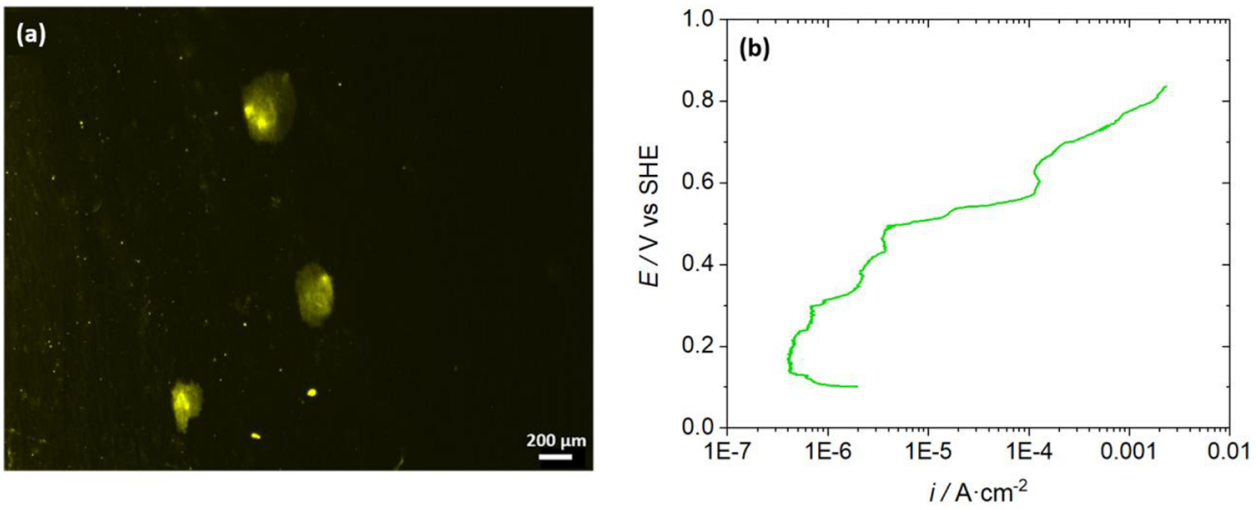

Figure 5. (a) Detection of sites showing corrosion on a 304 stainless steel substrate coated with the nanosensors/polymer composite. The sample was observed with a fluorescent microscope (CY3 filter) after being exposed to a $35 \mathrm{~g} \mathrm{~L}^{-1} \mathrm{NaCl}$ solution and subjected to a potentiodynamic polarization scan toward nobler potentials presented in panel (b), with a classical three-electrode system in order to initiate localized corrosion. Optically, no corrosion attack was yet visible.

large peaks caused by the diffusion of individual bright nanocontainers through the FCS observation volume were evident. On the trace measured after $4 \mathrm{~h}$, the peaks were much weaker, indicating that the nanocontainers lost their fluorescence brightness as a result of the release of the activated fluorescent molecules. In order to quantify this effect, we evaluated the fluorescent brightness of the nanocontainers (see Experimental Section) for all four studied dispersions and plotted it versus the time after mixing (Figure $4 \mathrm{~b}$ ). As can be seen for both strongly acidic dispersions $(\mathrm{pH}=2.9$ with or without ferric ions), a strong fluorescence brightness of the nanocontainers was observed immediately, followed by a rapid decrease in the first hours before reaching a plateau. As discussed above, this decrease was attributed to the diffusion of some of the activated fluorescent molecules from the capsules in the surrounding medium. A similar tendency for both solutions in the first hours showed that acidity was the main contribution to fluorescence in the first hours. Concerning the solution with a lower concentration of ferric ions $(0.05 \mathrm{mmol}$ $\left.\mathrm{L}^{-1}\right)$ and the corresponding $\mathrm{pH}$ solution $(\mathrm{pH}=5)$, the fluorescent brightness was much lower than the previous ones in the first hours. The fluorescence brightness of particles remained constant during the measurement for the solution without ferric ions at $\mathrm{pH}=5$, unlike the dispersions in the 0.05 mol L $\mathrm{L}^{-1}$ ferric solution, whose brightness increased with time. This could be attributed to the higher solubility of ferric ions in toluene compared to water solubility in toluene. Ferric ions can migrate through the pores of the silica capsule to the liquid core, forming a fluorescent complex with the dye inside the capsules. The FCS experiment results allow a better understanding of the mechanism of the nanosensor. At high concentration of ferric solution, which is naturally associated with low $\mathrm{pH}$ values, the nanosensor showed an instantaneous large brightness in the very first hours and then a partial release of some of the activated fluorescent molecules from the capsules into the surrounding areas, which remained fluorescent for longer times. The nanosensors are therefore suitable for the detection of early stages of corrosion, leading to convenient and accurate localization of the affected areas. The FCS results, together with fluorescence measurements of the complexed dye at different times (Figure S7), showed that the fluorescence signal gave semiquantitative information about the concentration of iron(III). The fluorescence intensity is indeed a complex interplay of the concentrations of dye and metal ions, time of equilibration for building the complex, and diffusion of the metal ion toward the dye. Clearly, the time scales under real conditions, i.e., when the nanocapsules are incorporated in coatings, will be significantly longer. However, the FCS studies are aimed at better understanding of the mechanism of the nanosensor, i.e., understanding the nature of the fluorescence changes observed with classical methods.

To further investigate the potential for early corrosion detection on a steel substrate, the behavior of a model composite coating upon corrosion was investigated. The coating was formed by the nanosensors incorporated in a poly(vinyl butyral-co-vinyl alcohol-co-vinyl acetate) matrix. This polymer was already used in previous studies as coating on zinc substrates to embed nanocontainers encapsulating corrosion inhibitors ${ }^{30}$ and self-healing agents. ${ }^{31}$ Moreover, it is considered as a model coating that was investigated for the inhibition of corrosion-driven underfilm delamination. ${ }^{32}$ Furthermore, it has good weatherability and barrier properties. ${ }^{33}$ Diffusion of the ions in the solid coating is expected to be very slow. Therefore, the coating will be active only if there is a contact or at least promiscuity between aqueous medium containing ions, either produced by condensation or from rain, and the nanosensors. This can be produced by mechanical damage of the coating or during the pitting corrosion process.

The composite coating was applied on a 304 stainless steel substrate that was previously mechanically polished. The coated sample was exposed to a potentiodynamic polarization scan to initiate corrosion at pinhole sites in the coating (Figure $5 b)$. Observation of the exposed area to corrosive solution with a fluorescent microscope allowed for clearly detecting areas where localized corrosion was initiated. In Figure 5a, one part of the coating covering the defect showed strong fluorescence intensity, due to the first steps of anodic dissolution of steel. Areas severely affected by anodic dissolution of metal substrate actually contain a higher amount of dissolved metallic cations and higher acidity. The nanosensors located close to these areas, i.e., inside the coating directly covering or close by, emitted a fluorescent signal of higher intensity. When excited with the appropriate light wavelength, regions already affected by the early stage of corrosion can be hence detected by fluorescence. Fluorescence in pits could still be observed several months after initiation of the pitting. Indeed, we believe that activated fluorescent molecules were partially released 
from the capsules into the surrounding areas, as shown by fluorescence spectroscopy measurements.

Metal alloys may produce metal cations that may interfere with the dye. Furthermore, iron(II) can be also produced during the corrosion process. Therefore, we investigated the selectivity of the sensor to the type of metal ions. For comparison, we measured the fluorescence intensity of dye in the presence of $\mathrm{Zn}(\mathrm{II}), \mathrm{Al}(\mathrm{III})$, and $\mathrm{Fe}(\mathrm{II})$ ions. $\mathrm{Fe}(\mathrm{II})$ is also complexed by the dye. However, the fluorescence intensity was much lower for $\mathrm{Fe}$ (II) (Figure S8a) compared to the fluorescence intensity with $\mathrm{Fe}$ (III) at the same concentrations (Figure S8b). Although $\mathrm{Fe}(\mathrm{II})$ ions would participate in the emitted signal, its contribution would be much lower compared to the contribution of $\mathrm{Fe}$ (III) ions. Interestingly, the fluorescence emission with $\mathrm{Zn}$ (II) ions is very weak (Figure S9a). On the contrary, the signal generated by the complexation with $\mathrm{Al}(\mathrm{III})$ ions is strong (Figure S9b). However, it does not reach the intensity provided by the complexation with $\mathrm{Fe}(\mathrm{III})$ ions (Figure S9c). An overall comparison for the different ions is depicted in Figure S10. These experiments infer one advantage and one limitation for the system. Indeed, it means that the system could be used for the early detection of corrosion of aluminum. However, it would be impossible to get completely quantitative feedback based on fluorescence signal because of the non-negligible contributions of $\mathrm{Fe}(\mathrm{II})$ and $\mathrm{Al}(\mathrm{III})$ to the emitted signal.

\section{CONCLUSIONS}

Nanosensors consisting of silica nanocapsules containing a dye were prepared by the miniemulsion process. The nanosensors were porous so that iron(III) ions can diffuse through the silica shell into the core to yield a signal due to chelation-enhanced fluorescence. The fluorescent intensity depended on the quantity of ferric ions in the direct vicinity of the capsules, allowing an observation of the occurrence of early stage corrosion in areas where rust was not yet clearly visible. The nanosensors were successfully embedded in a polymer matrix applied on a metal substrate. This composite coating was capable of early stage corrosion detection.

\section{ASSOCIATED CONTENT}

\section{S Supporting Information}

The Supporting Information is available free of charge on the ACS Publications website at DOI: 10.1021/acsanm.8b02045.

Photographs of dye solutions under sunlight and UV light, UV spectra, and fluorescence emission spectra of the dye and of nanocontainers encapsulating the dye, and temporal evolution of the fluorescence intensity (PDF)

\section{AUTHOR INFORMATION}

\section{Corresponding Authors}

*(K.L) E-mail: landfester@mpip-mainz.mpg.de.

*(D.C.) E-mail: daniel.crespy@vistec.ac.th.

\section{ORCID}

Matthias Uebel: 0000-0002-7825-7047

Kaloian Koynov: 0000-0002-4062-8834

Katharina Landfester: 0000-0001-9591-4638

Daniel Crespy: 0000-0002-6023-703X

\section{Author Contributions}

${ }^{\#}$ L.E. and S.S. contributed equally to this work.

\section{Notes}

The authors declare no competing financial interest.

\section{ACKNOWLEDGMENTS}

We are grateful for discussions with Elisa Campazzi, Professor Sebastien Touzain, and Professor Juan Creus. This work was partially supported by the Thailand Research Fund (Grant RSA6180063), the Office of Higher Education Commission of Thailand (OHEC), the DFG priority program SPP1568 "Design and Generic Principles of Self-Healing Materials", and the Vidyasirimedhi Institute of Science and Technology (VISTEC). R.J. appreciates the Postdoctoral Fellowship of the Vidyasirimedhi Institute of Science and Technology. Instrumental support from the Frontier Research Center (FRC) at VISTEC is also acknowledged.

\section{REFERENCES}

(1) Gotou, T.; Noda, M.; Tomiyama, T.; Sembokuya, H.; Kubouchi, M.; Tsuda, K. In Situ Health Monitoring of Corrosion Resistant Polymers Exposed to Alkaline Solutions Using $\mathrm{pH}$ Indicators. Sens. Actuators, B 2006, 119, 27-32.

(2) Dhole, G. S.; Gunasekaran, G.; Singh, S. K.; Vinjamur, M. Smart Corrosion Sensing Phenanthroline Modified Alkyd Coatings. Prog. Org. Coat. 2015, 89, 8-16.

(3) Dhole, G. S.; Gunasekaran, G.; Ghorpade, T.; Vinjamur, M. Smart Acrylic Coatings for Corrosion Detection. Prog. Org. Coat. 2017, 110, 140-149.

(4) Maia, F.; Tedim, J.; Bastos, A. C.; Ferreira, M. G. S.; Zheludkevich, M. L. Nanocontainer-based Corrosion Sensing Coating. Nanotechnology 2013, 24, 415502-415510.

(5) Maia, F.; Tedim, J.; Bastos, A. C.; Ferreira, M. G. S.; Zheludkevich, M. L. Active Sensing Coating for Early Detection of Corrosion Processes. RSC Adv. 2014, 4, 17780-17786.

(6) Zhang, J.; Frankel, G. S. Corrosion-Sensing Behavior of an Acrylic-based Coating System. Corrosion 1999, 55, 957-967.

(7) Liu, G.; Wheat, H. G. Use of a Fluorescent Indicator in Monitoring Underlying Corrosion on Coated Aluminum 2024-T4. J. Electrochem. Soc. 2009, 156, C160-C166.

(8) Johnson, R. E.; Agarwala, V. S. Using Fluorescent Compounds as Early Warning Detectors for Corrosion. Mater. Perform. 1994, 33, $25-29$.

(9) Alodan, M. A.; Smyrl, W. H. Detection of Localized Corrosion of Aluminum Alloys Using Fluorescence Microscopy. J. Electrochem. Soc. 1998, 145, 1571-1577.

(10) Warren-Smith, S. C.; Heng, S.; Ebendorff-Heidepriem, H.; Abell, A. D.; Monro, T. M. Fluorescence-based Aluminum Ion Sensing Using a Surface-functionalized Microstructured Optical Fiber. Langmuir 2011, 27, 5680-5685.

(11) Venancio, P. G.; Cottis, R. A.; Narayanaswamy, R.; Fernandes, J. C. S. Optical Sensors for Corrosion Detection in Airframes. Sens. Actuators, B 2013, 182, 774-781.

(12) Szunerits, S.; Walt, D. R. Aluminum Surface Corrosion and the Mechanism of Inhibitors Using $\mathrm{pH}$ and Metal Ion Selective Imaging Fiber Bundles. Anal. Chem. 2002, 74, 886-894.

(13) Nguyen, T. H.; Venugopala, T.; Chen, S.; Sun, T.; Grattan, K. T. V.; Taylor, S. E.; Basheer, P. A. M.; Long, A. E. Fluorescence Based Fibre Optic $\mathrm{pH}$ Sensor for the $\mathrm{pH}$ 10-13 Range Suitable for Corrosion Monitoring in Concrete Structures. Sens. Actuators, B 2014, 191, 498-507.

(14) Roshan, S.; Sarabi Dariani, A. A.; Mokhtari, J. Monitoring Underlying Epoxy-coated St-37 Corrosion via 8-Hydroxyquinoline as a Fluorescent Indicator. Appl. Surf. Sci. 2018, 440, 880-888.

(15) Augustyniak, A.; Tsavalas, J.; Ming, W. Early Detection of Steel Corrosion via "Turn-on" Fluorescence in Smart Epoxy Coatings. ACS Appl. Mater. Interfaces 2009, 1, 2618-2623. 
(16) Lee, T. H.; Song, Y. K.; Park, S. H.; Park, Y. I.; Noh, S. M.; Kim, J. C. Dual Stimuli Responsive Self-reporting Material for Chemical Reservoir Coating. Appl. Surf. Sci. 2018, 434, 1327-1335.

(17) Zhang, M.; Gao, Y.; Li, M.; Yu, M.; Li, F.; Li, L.; Zhu, M.; Zhang, J.; Yi, T.; Huang, C. A Selective Turn-on Fluorescent Sensor for $\mathrm{Fe}^{\mathrm{III}}$ and Application to Bioimaging. Tetrahedron Lett. 2007, 48, 3709-3712.

(18) Rigler, R.; Elson, E. S., Eds. Fluorescence Correlation Spectroscopy: Theory and Applications, 1st ed.; Springer-Verlag: Berlin, Heidelberg, New York, 2001.

(19) Vijayshankar, D.; Altin, A.; Merola, C.; Bashir, A.; Heinen, E.; Rohwerder, M. Probing the Buried Metal-Organic Coating Interfacial Reaction Kinetic Mechanisms by a Hydrogen Permeation Based Potentiometric Approach. J. Electrochem. Soc. 2016, 163, C778-C783.

(20) Fickert, J.; Rupper, P.; Graf, R.; Landfester, K.; Crespy, D. Design and Characterization of Functionalized Silica Nanocontainers for Self-healing Materials. J. Mater. Chem. 2012, 22, 2286-2291.

(21) Fickert, J.; Schaeffel, D.; Koynov, K.; Landfester, K.; Crespy, D. Silica Nanocapsules for Redox-responsive Delivery. Colloid Polym. Sci. 2014, 292, 251-255.

(22) Jiang, S.; Lv, L.; Li, Q.; Wang, J.; Landfester, K.; Crespy, D. Tailoring Nanoarchitectonics to Control the Release Profile of Payloads. Nanoscale 2016, 8, 11511-11517.

(23) Behzadi, S.; Steinmann, M.; Estupiñán, D.; Landfester, K.; Crespy, D. The Pro-active Payload Strategy Significantly Increases Selective Release from Mesoporous Nanocapsules. J. Controlled Release 2016, 242, 119-125.

(24) Jiang, S.; Ma, B. C.; Reinholz, J.; Li, Q.; Wang, J.; Zhang, K. A. I.; Landfester, K.; Crespy, D. Efficient Nanofibrous Membranes for Antibacterial Wound Dressing and UV Protection. ACS Appl. Mater. Interfaces 2016, 8, 29915-29922.

(25) Jiang, S.; Lieberwirth, I.; Landfester, K.; Muñoz-Espí, R.; Crespy, D. Nanofibrous Photocatalysts from Electrospun Nanocapsules. Nanotechnology 2017, 28, 405601-405609.

(26) Afaneh, A. T.; Schreckenbach, G. Fluorescence Enhancement/ Quenching Based on Metal Orbital Control: Computational Studies of a 6-Thienyllumazine-Based Mercury Sensor. J. Phys. Chem. A 2015, 119, 8106-8116.

(27) Frankel, G. S. Pitting Corrosion of Metals: A Review of the Critical Factors. J. Electrochem. Soc. 1998, 145, 2186-2198.

(28) Koynov, K.; Butt, H.-J. Fluorescence Correlation Spectroscopy in Colloid and Interface Science. Curr. Opin. Colloid Interface Sci. 2012, 17, 377-387.

(29) Schaeffel, D.; Staff, R. H.; Butt, H.-J.; Landfester, K.; Crespy, D.; Koynov, K. Fluorescence Correlation Spectroscopy Directly Monitors Coalescence During Nanoparticle Preparation. Nano Lett. 2012, 12, 6012-6017.

(30) Vimalanandan, A.; Lv, L. P.; Tran, T. H.; Landfester, K.; Crespy, D.; Rohwerder, M. Redox-responsive Self-healing for Corrosion Protection. Adv. Mater. 2013, 25, 6980-6984.

(31) Tran, T. H.; Vimalanandan, A.; Genchev, G.; Fickert, J.; Landfester, K.; Crespy, D.; Rohwerder, M. Regenerative Nano-hybrid Coating Tailored for Autonomous Corrosion Protection. Adv. Mater. 2015, 27, 3825-3830.

(32) Williams, G.; McMurray, H. N. Chromate Inhibition of Corrosion-driven Organic Coating Delamination Studied Using a Scanning Kelvin Probe Technique. J. Electrochem. Soc. 2001, 148, B377-B385

(33) Cambier, S. M.; Posner, R.; Frankel, G. S. Coating and Interface Degradation of Coated Steel, Part 1: Field Exposure. Electrochim. Acta 2014, 133, 30-39. 